\title{
Deviations of Steady States of the Traveling Wave to a Competition Diffusion System with Random Perturbation
}

\author{
Xiaorong Hu $\mathbf{H}^{1}$, Yanbin Tang² \\ ${ }^{1}$ Department of Mathematics, National University of Defense Technology, Changsha, China \\ ${ }^{2}$ School of Mathematics and Statistics, Huazhong University of Science and Technology, Wuhan, China \\ Email: 2472069301@qq.com, tangybhust@sina.com
}

Received 7 April 2015; accepted 11 May 2015; published 14 May 2015

Copyright (C 2015 by authors and Scientific Research Publishing Inc.

This work is licensed under the Creative Commons Attribution International License (CC BY). http://creativecommons.org/licenses/by/4.0/

cC) (i) Open Access

\section{Abstract}

This paper considers the asymptotic dynamics of steady states to the Lotka-Volterra competition diffusion systems with random perturbations by two-parameter white noise on the whole real line. By the fundamental solution of heat equation, we get the asymptotic fluctuating behaviors near the stable states respectively. That is, near the steady state $(u, v)=(0,1)$, the mean value $E u(x, t)$ is shifted above the equilibrium $u=0$ and $\operatorname{Ev}(x, t)$ is shifted below the equilibrium $v=1$. However, near the steady state $(u, v)=(1,0)$, the mean value $E u(x, t)$ is shifted below the equilibrium $u=1$ and $E v(x, t) \equiv 0$.

\section{Keywords}

Lotka-Volterra Competition Diffusion System, Random Perturbation, Two-Parameter White Noise

\section{Introduction}

Nonlinear reaction diffusion systems arise in several fields and have been studied by many authors (see [1] and the references therein). The theory of reaction diffusion waves began in the 1930s with the works by Fisher [2] [3], Kolmogorov, Petrovsky and Piskunov [4] on propagation of dominant gene and by Zeldovich et al. [5] in population dynamics, mathematical theory of combustion and chemical kinetics [6]. For example, H. C. Tuckwell [7] considered the general nonlinear reaction diffusion equation driven by two-parameter white noise

$$
u_{t}=u_{x x}+g(u)+\varepsilon W_{x t},
$$

How to cite this paper: Hu, X.R. and Tang, Y.B. (2015) Deviations of Steady States of the Traveling Wave to a Competition Diffusion System with Random Perturbation. Journal of Applied Mathematics and Physics, 3, 496-508.

http://dx.doi.org/10.4236/jamp.2015.35062 
where $\{W(x, t), x \in(-\infty,+\infty), t \geq 0\}$ was a standard two-parameter Wiener process, i.e., a Gaussian process with $E(W(x, t))=0, E(W(x, t) W(y, s))=\min \{x, y\} \min \{s, t\}, \varepsilon$ was a small real constant, and $g$ was a function at least twice differentiable at equilibrium.

At present time, it is a well developed area of research which includes qualitative properties of traveling wavefronts for many complex systems. Traveling waves are natural phenomena ubiquitously for reaction diffusion systems in many scientific areas, such as in biophysics, population genetics, mathematical ecology, chemistry, chemical physics and so on [8]-[14]. It is pretty well understood for a diffusing Lotka-Volterra (LV) system that there exist traveling wavefronts which propagate from an equilibrium to another one [15].

Consider the LV competition-diffusion system

$$
\left\{\begin{array}{l}
u_{t}=u_{x x}+(1-u-a v) u, \\
v_{t}=v_{x x}+r(1-b u-v) v,
\end{array}\right.
$$

where $u(x, t)>0, v(x, t)>0,(x, t) \in R \times R^{+}$, and $a, b, r$ are positive constants. We look for a monotone traveling wave solution of $(2)(u(x, t), v(x, t))=(U(\xi), V(\xi)), \xi=x+c t$, with wave speed $c$ under the boundary value conditions

$$
(U(-\infty), V(-\infty))=\left(u_{-}, v_{-}\right) ; \quad(U(+\infty), V(+\infty))=\left(u_{+}, v_{+}\right),
$$

where $\left(u_{-}, v_{-}\right)$and $\left(u_{+}, v_{+}\right)$are equilibria of (2):

$$
(0,0), \quad(1,0), \quad(0,1), \quad\left(\frac{1-a}{1-a b}, \frac{1-b}{1-a b}\right) .
$$

For $a<1, b<1$ or $a>1, b>1,\left(\frac{1-a}{1-a b}, \frac{1-b}{1-a b}\right)$ is a positive equilibrium. By the phase plane technique of ordinary differential equations in the first quadrant, we have the following cases for the system (see [3]).

1) Monostable case:

$(1,0)$ is stable; $(0,1)$ is unstable, $0<a<1<b$;

$(1,0)$ is unstable; $(0,1)$ is stable, $0<b<1<a$.

2) Coexistence case:

$\left(\frac{1-a}{1-a b}, \frac{1-b}{1-a b}\right)$ is stable, $a, b<1$.

3) Bistable case:

$(1,0)$ and $(0,1)$ are stable, $a, b>1$.

Traveling wavefronts of the system (2) have been studied very extensively. We refer readers to the references for traveling wave solutions connecting two equilibria.

1) Conley and Gardner [16] [17]:

$$
\left(u_{-}, v_{-}\right)=\left(\frac{1-a}{1-a b}, \frac{1-b}{1-a b}\right) \text { or }\left(u_{+}, v_{+}\right)=\left(\frac{1-a}{1-a b}, \frac{1-b}{1-a b}\right) ;
$$

2) Tang and Fife [18]:

$$
\left(u_{-}, v_{-}\right)=(0,0),\left(u_{+}, v_{+}\right)=\left(\frac{1-a}{1-a b}, \frac{1-b}{1-a b}\right)
$$

3) Kanel and Zhou [19]:

$$
\left(u_{-}, v_{-}\right)=\left(\frac{1-a}{1-a b}, \frac{1-b}{1-a b}\right),\left(u_{+}, v_{+}\right)=(1,0)
$$

4) Fei and Carr [15]:

$$
\left(u_{-}, v_{-}\right)=(0,1),\left(u_{+}, v_{+}\right)=(1,0) \text {. }
$$

For instance, we give some results on the traveling wave solutions of system (2). 
Theorem 1. [15] 1) If $0<a<1<b, 1-a \leq r(b-1)<1$, for the boundary value problem (2)-(3) with

$$
\left(u_{-}, v_{-}\right)=(0,1),\left(u_{+}, v_{+}\right)=(1,0) \text {, }
$$

there exist positive increasing traveling wavefronts $(U(x+c t), V(x+c t))$ with speed $c$ satisfying $c \geq 2 \sqrt{r(b-1)}$.

2) There do not exist traveling wavefront $(U(x+c t), V(x+c t))$ with speed $c$ satisfying

$$
c \leq\left[\frac{r}{2 \alpha}\left(\alpha^{2}-\beta^{2}\right)+r \beta \ln \frac{\beta}{\alpha}\right]^{1 / 2}
$$

where

$$
|\alpha|=\frac{b}{\rho}-1, \beta=\frac{b}{\rho}-b, \rho=\frac{1-r(b-1)}{a} .
$$

Theorem 2. [17] Let $\theta_{1}$ and $\theta_{2}$ be the velocities of the waves from $(0,1)$ to $(0,0)$ and from $(0,0)$ to $(1,0)$, respectively. Then if $\theta_{2}<\theta_{1}$, there is also a wave from $(0,1)$ to $(1,0)$.

In fact, under the conditions

$$
r=3 a, b=\frac{2}{3 a}+\frac{4}{3}, a \in(1,2),
$$

X. X. Bao and Z. C. Wang [20] gave explicit traveling wavefronts of the system (2) which connected the equilibria $(0,1)$ and $(1,0)$ :

$$
(u(x, t), v(x, t))=(U(\xi), V(\xi))=\left(\frac{1}{1+\mathrm{e}^{-\sqrt{\frac{a}{2} \xi}}}, \frac{1}{\left(1+\mathrm{e}^{\sqrt{\frac{a}{2} \xi}}\right)^{2}}\right),
$$

where $\xi=x+c t, c=\frac{2-a}{\sqrt{2 a}}$.

We know that in a linear system the noise does not affect the mean value at equilibrium; however, in a nonlinear system, the mean is displaced from an equilibrium. How can one describe this displaced mean value? H. C. Tuckwell [7] [21] gave a good idea. Using Green's functions, he described the nonlinear effects in white noise driven spatial diffusions. Following this idea, E. Z. Wu and Y. B. Tang [22] obtained the asymptotic fluctuating behaviors of the traveling wavefront to the Nagumo equation near two stable steady states.

In this paper, we are interested in calculating the statistical properties of the steady states of the LV competition-diffusion system (2) under the influence of random perturbations by two-parameter white noise $\varepsilon W_{x t}$ on the whole real line $\mathcal{R}$

$$
\left\{\begin{array}{l}
u_{t}=u_{x x}+(1-u-a v) u+\varepsilon W_{x t}, \quad x \in R, t>0, \\
v_{t}=v_{x x}+r(1-b u-v) v,
\end{array}\right.
$$

where $W(x, t)$ is a two-parameter Wiener process such that, formally,

$$
W(x, t)=\int_{0}^{t} \int_{0}^{x} w(y, s) \mathrm{d} y \mathrm{~d} s,
$$

where $w(x, t)$ stands for a generalized Gaussian random field with zero mean and correlation function

$$
E[w(x, t) w(y, s)]=\delta(x-y) \delta(t-s) .
$$

The initial condition to (8) is $(u(x, 0), v(x, 0))=\left(u_{0}, v_{0}\right)$ with probability one, and $\left(u_{0}, v_{0}\right)$ is one of the equilibria $(0,1)$ and $(1,0)$, and the boundary conditions of the traveling wavefront are $(U(-\infty), V(-\infty))=(0,1)$, $(U(+\infty), V(+\infty))=(1,0), \quad r, a, b$ are positive constants.

We present asymptotic representations of steady states of the LV competition diffusion system that it is 
randomly perturbed by two-parameter white noise $W_{x t}$ on the whole real line. For a traveling wavefront connecting two stable equilibria $(u=0, v=1)$ and $(u=1, v=0)$ of LV competition diffusion system, we first derive asymptotic representations of solutions near the steady states as $t \rightarrow+\infty$. Then by the fundamental solution of heat equation on the whole real line, we get the asymptotic fluctuating behaviors of steady states near the stable states respectively. That is, near the steady state $(u, v)=(0,1)$, the mean value $E u(x, t)$ is shifted above the equilibrium $u=0$ and $E v(x, t)$ is shifted below the equilibrium $v=1$. However, near the steady state $(u, v)=(1,0)$, the mean value $E u(x, t)$ is shifted below the equilibrium $u=1$ and $E v(x, t) \equiv 0$ is not affected by the noise perturbation.

\section{Random Perturbations on a Stationary State}

For $\varepsilon=0$, under the conditions (6), the system (2) has a monotone traveling wave solution connecting the two stable states $(0,1)$ and $(1,0)$. Let $\left(u_{0}, v_{0}\right)$ be an equilibrium of (2), i.e., $\left(u_{0}, v_{0}\right)=(0,1)$ or $\left(u_{0}, v_{0}\right)=(1,0)$.

We write the solution of the system (2) as

$$
\left(\begin{array}{l}
u(x, t) \\
v(x, t)
\end{array}\right)=\left(\begin{array}{l}
u_{0} \\
v_{0}
\end{array}\right)+\sum_{k=1}^{\infty} \varepsilon^{k}\left(\begin{array}{l}
u_{k} \\
v_{k}
\end{array}\right),\left(\begin{array}{l}
u(x, 0) \\
v(x, 0)
\end{array}\right)=\left(\begin{array}{l}
u_{0} \\
v_{0}
\end{array}\right)
$$

and rewrite the system (8) in the following form

$$
\left(\begin{array}{l}
u \\
v
\end{array}\right)_{t}=\left(\begin{array}{l}
u \\
v
\end{array}\right)_{x x}+\boldsymbol{A}\left(u_{0}, v_{0}\right)\left(\begin{array}{l}
u-u_{0} \\
v-v_{0}
\end{array}\right)-\boldsymbol{N}\left(u-u_{0}, v-v_{0}\right)+\varepsilon\left(\begin{array}{l}
1 \\
0
\end{array}\right) W_{x t},
$$

where

$$
\begin{gathered}
\boldsymbol{A}\left(u_{0}, v_{0}\right)=\left(\begin{array}{cc}
1-2 u_{0}-a v_{0} & -a u_{0} \\
-b r v_{0} & r-b r u_{0}-2 r v_{0}
\end{array}\right), \\
\boldsymbol{N}\left(u-u_{0}, v-v_{0}\right)=\left(\begin{array}{c}
\left(u-u_{0}\right)^{2}+a\left(u-u_{0}\right)\left(v-v_{0}\right) \\
b r\left(u-u_{0}\right)\left(v-v_{0}\right)+r\left(v-v_{0}\right)^{2}
\end{array}\right) .
\end{gathered}
$$

We put (11) into (12). Equating coefficients of powers of $\varepsilon$, we get the first two terms of a sequence of linear stochastic partial differential equations (SPDEs)

$$
\begin{aligned}
& \left(\begin{array}{l}
u_{1}(x, t) \\
v_{1}(x, t)
\end{array}\right)_{t}=\left(\begin{array}{l}
u_{1}(x, t) \\
v_{1}(x, t)
\end{array}\right)_{x x}+\boldsymbol{A}\left(u_{0}, v_{0}\right)\left(\begin{array}{l}
u_{1}(x, t) \\
v_{1}(x, t)
\end{array}\right)+\left(\begin{array}{l}
1 \\
0
\end{array}\right) W_{x t}, \\
& \left(\begin{array}{l}
u_{1}(x, 0) \\
v_{1}(x, 0)
\end{array}\right)=\left(\begin{array}{l}
0 \\
0
\end{array}\right) ; \\
& \left(\begin{array}{l}
u_{2}(x, t) \\
v_{2}(x, t)
\end{array}\right)_{t}=\left(\begin{array}{l}
u_{2}(x, t) \\
v_{2}(x, t)
\end{array}\right)_{x x}+A\left(u_{0}, v_{0}\right)\left(\begin{array}{l}
u_{2}(x, t) \\
v_{2}(x, t)
\end{array}\right)-N\left(u_{1}(x, t), v_{1}(x, t)\right), \\
& \left(\begin{array}{l}
u_{2}(x, 0) \\
v_{2}(x, 0)
\end{array}\right)=\left(\begin{array}{l}
0 \\
0
\end{array}\right) .
\end{aligned}
$$

As we know, the fundamental solution of the deterministic linear system

$$
\boldsymbol{u}_{t}(x, t)=\boldsymbol{u}_{x x}(x, t)+\boldsymbol{A u}(x, t)
$$

is

$$
\tilde{G}(x, t)=\mathrm{e}^{A t} G(x, t), G(x, t)=\frac{1}{\sqrt{4 \pi t}} \mathrm{e}^{-\frac{x^{2}}{4 t}}
$$

where $G(x, t)$ is the Green's function of the heat equation $u_{t}=u_{x x}$. It is easy to check that 


$$
\int_{-\infty}^{+\infty} G(x-y, t) \mathrm{d} y=1, \int_{-\infty}^{+\infty} G^{2}(x-y, t) \mathrm{d} y=\frac{1}{\sqrt{8 \pi t}} .
$$

From the sequence of linear SPDEs we have the solutions of initial value problems (15) and (16), respectively

$$
\begin{gathered}
\left(\begin{array}{l}
u_{1}(x, t) \\
v_{1}(x, t)
\end{array}\right)=\int_{0}^{t} \int_{-\infty}^{+\infty} G(x-y, t-s) \mathrm{e}^{A(t-s)}\left(\begin{array}{l}
1 \\
0
\end{array}\right) w(\mathrm{~d} y, \mathrm{~d} s) ; \\
\left(\begin{array}{l}
u_{2}(x, t) \\
v_{2}(x, t)
\end{array}\right)=-\int_{0}^{t} \int_{-\infty}^{+\infty} G(x-y, t-s) \mathrm{e}^{A(t-s)} N\left(u_{1}, v_{1}\right) \mathrm{d} y \mathrm{~d} s .
\end{gathered}
$$

According to the zero-mean property of Itô integral we have

$$
\begin{gathered}
E\left(\begin{array}{l}
u_{1}(x, t) \\
v_{1}(x, t)
\end{array}\right)=\left(\begin{array}{l}
0 \\
0
\end{array}\right) ; \\
E\left(\begin{array}{l}
u_{2}(x, t) \\
v_{2}(x, t)
\end{array}\right)=-\int_{0}^{t} \int_{-\infty}^{+\infty} G(\cdot, \cdot) \mathrm{e}^{A(t-s)}\left(\begin{array}{c}
E\left[u_{1}^{2}+a u_{1} v_{1}\right] \\
E\left[b r u_{1} v_{1}+r v_{1}^{2}\right]
\end{array}\right) \text { dyds. }
\end{gathered}
$$

These give the expectation of stochastic process $u(x, t)$ to order $\varepsilon^{2}$ near the equilibrium $\left(u_{0}, v_{0}\right)$

$$
E\left(\begin{array}{l}
u(x, t) \\
v(x, t)
\end{array}\right)=\left(\begin{array}{l}
u_{0} \\
v_{0}
\end{array}\right)+\varepsilon^{2} E\left(\begin{array}{l}
u_{2}(x, t) \\
v_{2}(x, t)
\end{array}\right)+o\left(\varepsilon^{2}\right) .
$$

\section{Asymptotic Random Perturbations on the Left Stable State}

The equilibrium $(0,1)$ is the left stable state of the traveling wavefront of (2), i.e., $(U(-\infty), V(-\infty))=(0,1)$. Now we consider the equilibrium $\left(u_{0}, v_{0}\right)=(0,1)$. Under the condition (6), the linearized matrix of $(2)$ at $(0,1)$ is

$$
A(0,1)=\left(\begin{array}{cc}
1-a & 0 \\
-b r & -r
\end{array}\right),
$$

it has two negative eigenvalues $\lambda_{1}=1-a<0, \lambda_{2}=-r<0$, and there is an invertible matrix

$$
\boldsymbol{P}=\left(\begin{array}{cc}
1 & 0 \\
-2 & 1
\end{array}\right)
$$

such that

$$
\boldsymbol{P}^{-1} \boldsymbol{A}(0,1) \boldsymbol{P}=\left(\begin{array}{cc}
1-a & 0 \\
0 & -r
\end{array}\right)
$$

thus

$$
\mathrm{e}^{A t}=\boldsymbol{P}\left(\begin{array}{cc}
\mathrm{e}^{(1-a) t} & 0 \\
0 & \mathrm{e}^{-r t}
\end{array}\right) \boldsymbol{P}^{-1}=\left(\begin{array}{cc}
\mathrm{e}^{(1-a) t} & 0 \\
-2\left[\mathrm{e}^{(1-a) t}-\mathrm{e}^{-r t}\right] & \mathrm{e}^{-r t}
\end{array}\right)
$$

Therefore, the solution of (15) is

$$
\begin{gathered}
\left(\begin{array}{l}
u_{1}(x, t) \\
v_{1}(x, t)
\end{array}\right)=\int_{0}^{t} \int_{-\infty}^{+\infty} G(x-y, t-s) \mathrm{e}^{\mathrm{A}(0,1)(t-s)}\left(\begin{array}{l}
1 \\
0
\end{array}\right) w(\mathrm{~d} y, \mathrm{~d} s) \\
=\int_{0}^{t} \int_{-\infty}^{+\infty} G(x-y, t-s) M_{1} w(\mathrm{~d} y, \mathrm{~d} s), \\
M_{1}=\mathrm{e}^{A(0,1)(t-s)}\left(\begin{array}{l}
1 \\
0
\end{array}\right)=\left(\begin{array}{c}
\mathrm{e}^{(1-a)(t-s)} \\
\left.-2\left[\mathrm{e}^{(1-a)(t-s)}-\mathrm{e}^{-r(t-s)}\right]\right) ;
\end{array}\right.
\end{gathered}
$$




$$
\begin{gathered}
\left(\begin{array}{l}
u_{2}(x, t) \\
v_{2}(x, t)
\end{array}\right)=-\int_{0}^{t} \int_{-\infty}^{+\infty} G(x-y, t-s) \mathrm{e}^{A(t-s)} N\left(u_{1}(y, s), v_{1}(y, s)\right) \mathrm{d} y \mathrm{~d} s \\
=-\int_{0}^{t} \int_{-\infty}^{+\infty} G(x-y, t-s) M_{2}(y, s) \mathrm{d} y \mathrm{~d} s, \\
M_{2}=\left(\begin{array}{c}
\mathrm{e}^{(1-a)(t-s)}\left(u_{1}^{2}+a u_{1} v_{1}\right) \\
-2\left[\mathrm{e}^{(1-a)(t-s)}-\mathrm{e}^{-r(t-s)}\right]\left(u_{1}^{2}+a u_{1} v_{1}\right)+\mathrm{e}^{-r(t-s)}\left(b r u_{1} v_{1}+r v_{1}^{2}\right)
\end{array}\right) .
\end{gathered}
$$

In order to compute the expectations $E u_{2}(x, t)$ and $E v_{2}(x, t)$, we first calculate the following quantities.

$$
\begin{gathered}
E u_{1}^{2}(x, t)=E\left(\int_{0}^{t} \int_{-\infty}^{+\infty} G(x-y, t-s) \mathrm{e}^{(1-a)(t-s)} w(\mathrm{~d} y, \mathrm{~d} s)\right)^{2} \\
=\int_{0}^{t} \int_{-\infty}^{+\infty} G^{2}(x-y, t-s) \mathrm{e}^{2(1-a)(t-s)} \mathrm{d} y \mathrm{~d} s \\
=\int_{0}^{t} \frac{1}{\sqrt{8 \pi(t-s)}} \mathrm{e}^{2(1-a)(t-s)} \mathrm{d} s \\
=\int_{0}^{t} \frac{1}{\sqrt{8 \pi s}} \mathrm{e}^{2(1-a) s} \mathrm{~d} s . \\
E v_{1}^{2}(x, t)=E\left(-2 \int_{0}^{t} \int_{-\infty}^{+\infty} G(x-y, t-s)\left[\mathrm{e}^{(1-a)(t-s)}-\mathrm{e}^{-r(t-s)}\right] w(\mathrm{~d} y, \mathrm{~d} s)\right)^{2} \\
=4 \int_{0}^{t} \int_{-\infty}^{+\infty} G^{2}(x-y, t-s)\left[\mathrm{e}^{(1-a)(t-s)}-\mathrm{e}^{-r(t-s)}\right]^{2} \mathrm{~d} y \mathrm{~d} s \\
=4 \int_{0}^{t} \frac{1}{\sqrt{8 \pi(t-s)}}\left[\mathrm{e}^{(1-a)(t-s)}-\mathrm{e}^{-r(t-s)}\right]^{2} \mathrm{~d} s \\
=4 \int_{0}^{t} \frac{1}{\sqrt{8 \pi s}}\left[\mathrm{e}^{(1-a) s}-\mathrm{e}^{-r s}\right]^{2} \mathrm{~d} s .
\end{gathered}
$$

Since $E\left(u_{1}^{2}+a u_{1} v_{1}\right)=E\left(u_{1}+\frac{a}{2} v_{1}\right)^{2}-\frac{a^{2}}{4} E v_{1}^{2}$, and

$$
\begin{aligned}
E\left(u_{1}+\frac{a}{2} v_{1}\right)^{2} & =E\left(\int_{0}^{t} \int_{-\infty}^{+\infty} G(x-y, t-s)\left[(1-a) \mathrm{e}^{(1-a)(t-s)}+a \mathrm{e}^{-r(t-s)}\right] w(\mathrm{~d} y, \mathrm{~d} s)\right)^{2} \\
& =\int_{0}^{t} \int_{-\infty}^{+\infty} G^{2}(x-y, t-s)\left[(1-a) \mathrm{e}^{(1-a)(t-s)}+a \mathrm{e}^{-r(t-s)}\right]^{2} \mathrm{~d} y \mathrm{~d} s \\
& =\int_{0}^{t} \frac{1}{\sqrt{8 \pi(t-s)}}\left[(1-a) \mathrm{e}^{(1-a)(t-s)}+a \mathrm{e}^{-r(t-s)}\right]^{2} \mathrm{~d} s \\
& =\int_{0}^{t} \frac{1}{\sqrt{8 \pi s}}\left[(1-a) \mathrm{e}^{(1-a) s}+a \mathrm{e}^{-r s}\right]^{2} \mathrm{~d} s
\end{aligned}
$$

so we have

$$
\begin{aligned}
E\left(u_{1}^{2}+a u_{1} v_{1}\right) & =\int_{0}^{t} \frac{1}{\sqrt{8 \pi s}}\left\{\left[(1-a) \mathrm{e}^{(1-a) s}+a \mathrm{e}^{-r s}\right]^{2}-a^{2}\left[\mathrm{e}^{(1-a) s}-\mathrm{e}^{-r s}\right]^{2}\right\} \mathrm{d} s \\
& =\int_{0}^{t} \frac{1}{\sqrt{8 \pi s}}\left[(1-2 a) \mathrm{e}^{2(1-a) s}+2 a \mathrm{e}^{(1-a-r) s}\right] \mathrm{d} s .
\end{aligned}
$$

Since $E\left(b u_{1} v_{1}+v_{1}^{2}\right)=E\left(\frac{b}{2} u_{1}+v_{1}\right)^{2}-\frac{b^{2}}{4} E u_{1}^{2}$, and 


$$
\begin{aligned}
E\left(\frac{b}{2} u_{1}+v_{1}\right)^{2} & =E\left(\int_{0}^{t} \int_{-\infty}^{+\infty} G(x-y, t-s)\left[\left(\frac{b}{2}-2\right) \mathrm{e}^{(1-a)(t-s)}+2 \mathrm{e}^{-r(t-s)}\right] w(\mathrm{~d} y, \mathrm{~d} s)\right)^{2} \\
& =\int_{0}^{t} \int_{-\infty}^{+\infty} G^{2}(x-y, t-s)\left[\left(\frac{b}{2}-2\right) \mathrm{e}^{(1-a)(t-s)}+2 \mathrm{e}^{-r(t-s)}\right]^{2} \mathrm{~d} y \mathrm{~d} s \\
& =\int_{0}^{t} \frac{1}{\sqrt{8 \pi(t-s)}}\left[\left(\frac{b}{2}-2\right) \mathrm{e}^{(1-a)(t-s)}+2 \mathrm{e}^{-r(t-s)}\right]^{2} \mathrm{~d} s \\
& =\int_{0}^{t} \frac{1}{\sqrt{8 \pi s}}\left[\left(\frac{b}{2}-2\right) \mathrm{e}^{(1-a) s}+2 \mathrm{e}^{-r s}\right]^{2} \mathrm{~d} s,
\end{aligned}
$$

so we have

$$
\begin{aligned}
E\left(b u_{1} v_{1}+v_{1}^{2}\right) & =\int_{0}^{t} \frac{1}{\sqrt{8 \pi s}}\left\{\left[\left(\frac{b}{2}-2\right) \mathrm{e}^{(1-a) s}+2 \mathrm{e}^{-r s}\right]^{2}-\frac{a^{2}}{4} \mathrm{e}^{2(1-a) s}\right\} \mathrm{d} s \\
& =\int_{0}^{t} \frac{(a-1) \mathrm{e}^{2(1-a) s}-(a-1+r) \mathrm{e}^{(1-a-r) s}+r \mathrm{e}^{-2 r s}}{\sqrt{8 \pi s} r / 4} \mathrm{ds} .
\end{aligned}
$$

Therefore, we get

$$
\begin{aligned}
E u_{2}(x, t)= & -\int_{0}^{t} \int_{-\infty}^{+\infty} G(x-y, t-s) \mathrm{e}^{(1-a)(t-s)} E\left(u_{1}^{2}+a u_{1} v_{1}\right)(y, s) \mathrm{d} y \mathrm{~d} s \\
= & -\int_{0}^{t} \mathrm{e}^{(1-a)(t-s)} E\left(u_{1}^{2}+a u_{1} v_{1}\right)(y, s) \mathrm{d} s \\
= & -\int_{0}^{t} \mathrm{e}^{(1-a)(t-s)} \int_{0}^{s} \frac{1}{\sqrt{8 \pi \tau}}\left[(1-2 a) \mathrm{e}^{2(1-a) \tau}+2 a \mathrm{e}^{(1-a-r) \tau}\right] \mathrm{d} \tau \mathrm{d} s . \\
E v_{2}(x, t)= & 2 \int_{0}^{t} \int_{-\infty}^{+\infty} G(x-y, t-s) \mathrm{e}^{(1-a)(t-s)} E\left(u_{1}^{2}+a u_{1} v_{1}\right)(y, s) \mathrm{d} y \mathrm{~d} s \\
& -2 \int_{0}^{t} \int_{-\infty}^{+\infty} G(x-y, t-s) \mathrm{e}^{-r(t-s)} E\left(u_{1}^{2}+a u_{1} v_{1}\right)(y, s) \mathrm{d} y \mathrm{~d} s \\
& -\int_{0}^{t} \int_{-\infty}^{+\infty} G(x-y, t-s) \mathrm{e}^{-r(t-s)} E\left(b r u_{1} v_{1}+r v_{1}^{2}\right)(y, s) \mathrm{d} y \mathrm{~d} s \\
= & 2 \int_{0}^{t} \mathrm{e}^{(1-a)(t-s)} E\left(u_{1}^{2}+a u_{1} v_{1}\right)(y, s) \mathrm{d} s \\
& -2 \int_{0}^{t} \mathrm{e}^{-r(t-s)} E\left(u_{1}^{2}+a u_{1} v_{1}\right)(y, s) \mathrm{d} s \\
& -\int_{0}^{t} \mathrm{e}^{-r(t-s)} E\left(b r u_{1} v_{1}+r v_{1}^{2}\right)(y, s) \mathrm{d} s,
\end{aligned}
$$

that is,

$$
\begin{aligned}
E v_{2}(x, t)= & 2 \int_{0}^{t} \mathrm{e}^{(1-a)(t-s)} \int_{0}^{s} \frac{1}{\sqrt{8 \pi \tau}}\left[(1-2 a) \mathrm{e}^{2(1-a) \tau}+2 a \mathrm{e}^{(1-a-r) \tau}\right] \mathrm{d} \tau \mathrm{d} s \\
& -2 \int_{0}^{t} \mathrm{e}^{-r(t-s)} \int_{0}^{s} \frac{1}{\sqrt{8 \pi \tau}}\left[(1-2 a) \mathrm{e}^{2(1-a) \tau}+2 a \mathrm{e}^{(1-a-r) \tau}\right] \mathrm{d} \tau \mathrm{d} s \\
& -4 \int_{0}^{t} \mathrm{e}^{-r(t-s)} \int_{0}^{s} \frac{(a-1) \mathrm{e}^{2(1-a) \tau}-(a-1+r) \mathrm{e}^{(1-a-r) \tau}+r \mathrm{e}^{-2 r \tau}}{\sqrt{8 \pi \tau}} \mathrm{d} \tau \mathrm{d} s .
\end{aligned}
$$

As complexity of the formula of expectation $E\left[u_{2}(x, t)\right]$ and $E\left[v_{2}(x, t)\right]$, it is very difficult to determine the signs of $E\left[u_{2}(x, t)\right]$ and $E\left[v_{2}(x, t)\right]$ respectively, we just consider the asymptotic behavior of $E\left[u_{2}(x, t)\right]$ and $E\left[v_{2}(x, t)\right]$ as $t \rightarrow+\infty$.

By the formula 


$$
\int_{0}^{+\infty} \frac{1}{\sqrt{s}} \mathrm{e}^{-\alpha s} \mathrm{~d} s=\sqrt{\frac{\pi}{\alpha}} \quad(\alpha>0),
$$

and l'Hôpital's rule, we have

$$
\begin{aligned}
\lim _{t \rightarrow+\infty} E u_{2} & =-\lim _{t \rightarrow+\infty} \int_{0}^{t} \mathrm{e}^{(1-a)(t-s)} \int_{0}^{s} \frac{1}{\sqrt{8 \pi \tau}}\left[(1-2 a) \mathrm{e}^{2(1-a) \tau}+2 a \mathrm{e}^{(1-a-r) \tau}\right] \mathrm{d} \tau \mathrm{d} s \\
& =-\lim _{t \rightarrow+\infty} \frac{\int_{0}^{t} \mathrm{e}^{-(1-a) s} \int_{0}^{s} \frac{1}{\sqrt{8 \pi \tau}}\left[(1-2 a) \mathrm{e}^{2(1-a) \tau}+2 a \mathrm{e}^{(1-a-r) \tau}\right] \mathrm{d} \tau \mathrm{d} s}{\mathrm{e}^{-(1-a) t}} \\
& =\frac{-1}{a-1} \int_{0}^{+\infty} \frac{1}{\sqrt{8 \pi \tau}}\left[(1-2 a) \mathrm{e}^{2(1-a) \tau}+2 a \mathrm{e}^{(1-a-r) \tau}\right] \mathrm{d} \tau \\
& =\frac{-1}{(a-1) \sqrt{8 \pi}}\left[(1-2 a) \sqrt{\frac{\pi}{2(a-1)}}+2 a \sqrt{\frac{\pi}{r-1+a}}\right] \\
& =\frac{1}{(a-1) \sqrt{8}}\left[\frac{2 a-1}{\sqrt{2 a-2}}-\frac{2 a}{\sqrt{4 a-1}}\right] \\
& \left.=\frac{8 a^{3}-12 a^{2}+8 a-1}{\sqrt{8}(a-1) \sqrt{2 a-2} \sqrt{4 a-1}[(2 a-1) \sqrt{4 a-1}+2 a \sqrt{2 a-2}}\right]
\end{aligned}
$$

Denote $f(a)=8 a^{3}-12 a^{2}+8 a-1, a \in(1,2)$, since

$$
\begin{aligned}
f^{\prime}(a) & =24 a^{2}-24 a+8 \\
& =24\left(a-\frac{1}{2}\right)^{2}+2>0,
\end{aligned}
$$

and $f(1)=3>0$, then $f(a)>0, a \in(1,2)$, therefore

$$
\lim _{t \rightarrow+\infty} E u_{2}>0 \text {. }
$$

Similarly, we have

$$
\begin{aligned}
\lim _{t \rightarrow+\infty} E v_{2}(x, t)= & 2 \lim _{t \rightarrow+\infty} \int_{0}^{t} \mathrm{e}^{(1-a)(t-s)} \int_{0}^{s} \frac{1}{\sqrt{8 \pi \tau}}\left[(1-2 a) \mathrm{e}^{2(1-a) \tau}+2 a \mathrm{e}^{(1-a-r) \tau}\right] \mathrm{d} \tau \mathrm{d} s \\
& -2 \lim _{t \rightarrow+\infty} \int_{0}^{t} \mathrm{e}^{-r(t-s)} \int_{0}^{s} \frac{1}{\sqrt{8 \pi \tau}}\left[(1-2 a) \mathrm{e}^{2(1-a) \tau}+2 a \mathrm{e}^{(1-a-r) \tau}\right] \mathrm{d} \tau \mathrm{d} s \\
& -4 \lim _{t \rightarrow+\infty} \int_{0}^{t} \int_{0}^{s} \frac{(a-1) \mathrm{e}^{2(1-a) \tau}-(a-1+r) \mathrm{e}^{(1-a-r) \tau}+r \mathrm{e}^{-2 r \tau}}{\mathrm{e}^{r(t-s)} \sqrt{8 \pi \tau}} \mathrm{d} \tau \mathrm{d} s \\
= & 2 \lim _{t \rightarrow+\infty} \frac{\int_{0}^{t} \mathrm{e}^{-(1-a) s} \int_{0}^{s} \frac{1}{\sqrt{8 \pi \tau}}\left[(1-2 a) \mathrm{e}^{2(1-a) \tau}+2 a \mathrm{e}^{(1-a-r) \tau}\right] \mathrm{d} \tau \mathrm{d} s}{\mathrm{e}^{-(1-a) t}} \\
& -2 \lim _{t \rightarrow+\infty} \frac{\int_{0}^{t} \mathrm{e}^{r s} \int_{0}^{s} \frac{1}{\sqrt{8 \pi \tau}}\left[(1-2 a) \mathrm{e}^{2(1-a) \tau}+2 a \mathrm{e}^{(1-a-r) \tau}\right] \mathrm{d} \tau \mathrm{d} s}{\mathrm{e}^{r t}} \\
& -4 \lim _{t \rightarrow+\infty} \frac{\int_{0}^{t} \int_{0}^{s} \frac{\mathrm{e}^{r s}}{\sqrt{8 \pi \tau}}\left[(a-1) \mathrm{e}^{2(1-a) \tau}-(a-1+r) \mathrm{e}^{(1-a-r) \tau}+r \mathrm{e}^{-2 r \tau}\right] \mathrm{d} \tau \mathrm{d} s}{\mathrm{e}^{r t}},
\end{aligned}
$$

calculating the limits we have 


$$
\begin{aligned}
\lim _{t \rightarrow+\infty} E v_{2}(x, t)= & \frac{2}{a-1} \int_{0}^{+\infty} \frac{1}{\sqrt{8 \pi \tau}}\left[(1-2 a) \mathrm{e}^{2(1-a) \tau}+2 a \mathrm{e}^{(1-a-r) \tau}\right] \mathrm{d} \tau-\frac{2}{r} \int_{0}^{+\infty} \frac{1}{\sqrt{8 \pi \tau}}\left[(1-2 a) \mathrm{e}^{2(1-a) \tau}\right. \\
& \left.+2 a \mathrm{e}^{(1-a-r) \tau}\right] \mathrm{d} \tau-\frac{4}{r} \int_{0}^{+\infty} \frac{1}{\sqrt{8 \pi \tau}}\left[(a-1) \mathrm{e}^{2(1-a) \tau}-(a-1+r) \mathrm{e}^{(1-a-r) \tau}+r \mathrm{e}^{-2 r \tau}\right] \mathrm{d} \tau \\
= & \frac{2}{(a-1) \sqrt{8 \pi}}\left[(1-2 a) \sqrt{\frac{\pi}{2(a-1)}}+2 a \sqrt{\frac{\pi}{r+a-1}}\right]-\frac{2}{r \sqrt{8 \pi}}\left[(1-2 a) \sqrt{\frac{\pi}{2(a-1)}}\right. \\
& \left.+2 a \sqrt{\frac{\pi}{r+a-1}}\right]-\frac{4}{r \sqrt{8 \pi}}\left[(a-1) \sqrt{\frac{\pi}{2(a-1)}}-(a-1+r) \sqrt{\frac{\pi}{a-1+r}}+r \sqrt{\frac{\pi}{2 r}}\right],
\end{aligned}
$$

as $r=3 a$ in (6), we have

$$
\begin{aligned}
\lim _{t \rightarrow+\infty} E v_{2}(x, t)= & \frac{1}{a-1}\left[\frac{\sqrt{2} a}{\sqrt{4 a-1}}-\frac{2 a-1}{2 \sqrt{a-1}}\right]-\frac{1}{3 a}\left[\frac{\sqrt{2 a}}{\sqrt{4 a-1}}-\frac{2 a-1}{2 \sqrt{a-1}}\right]-\frac{1}{3 a}[\sqrt{a-1}-\sqrt{8 a-2}+\sqrt{3 a}] \\
= & \frac{1}{a-1}\left[\frac{\sqrt{2} a}{\sqrt{4 a-1}}-\frac{2 a-1}{2 \sqrt{a-1}}\right]+\frac{1}{3 a}\left[\frac{\sqrt{2}(3 a-1)}{\sqrt{4 a-1}}+\frac{1}{2 \sqrt{a-1}}-\sqrt{3 a}\right] \\
= & \frac{1}{3 a}\left[\frac{1}{2 \sqrt{a-1}}+\frac{\sqrt{2}(2 a-1)}{\sqrt{4 a-1}}-\sqrt{3 a}\right]+\frac{1}{a-1}\left[\frac{\sqrt{2} a}{\sqrt{4 a-1}}-\frac{2 a-1}{2 \sqrt{a-1}}+\frac{1}{3 a} \frac{\sqrt{2 a(a-1)}}{\sqrt{4 a-1}}\right] \\
= & \frac{1}{3 a}\left[\frac{1}{2 \sqrt{a-1}}-\frac{\sqrt{2}}{2 \sqrt{4 a-1}}+\frac{\sqrt{2} \sqrt{4 a-1}}{2}-\sqrt{3 a}\right]+\frac{1}{a-1}\left[\frac{\sqrt{2} \sqrt{4 a-1}}{3}-\frac{2 a-1}{2 \sqrt{a-1}}\right] \\
= & \frac{1}{3 a}\left[\frac{\sqrt{4 a-1}-\sqrt{2} \sqrt{a-1}}{2 \sqrt{a-1} \sqrt{4 a-1}}+\frac{2(4 a-1)}{2[\sqrt{2} \sqrt{4 a-1}+2 \sqrt{3 a}]}\right]+\frac{2 \sqrt{2} \sqrt{4 a-1} \sqrt{a-1}-3(2 a-1)}{6(a-1) \sqrt{a-1}} \\
= & \frac{2 a+1}{6 a \sqrt{a-1} \sqrt{4 a-1}[\sqrt{4 a-1}+\sqrt{2 a-2}]}-\frac{2 a+1}{3 a[\sqrt{8 a-2}+\sqrt{12 a}]} \\
& +\frac{(2 a+1)^{2}}{6(a-1) \sqrt{a-1}[2 \sqrt{2} \sqrt{4 a-1} \sqrt{a-1}+3(2 a-1)},
\end{aligned}
$$

that is,

$$
\begin{aligned}
\lim _{t \rightarrow+\infty} E v_{2}(x, t)= & -\frac{2 a+1}{3 a[\sqrt{8 a-2}+\sqrt{12 a}]}-\frac{2 a+1}{6 \sqrt{a-1}}\left\{\frac{2 a+1}{(a-1)[2 \sqrt{4 a-1} \sqrt{2 a-2}+6 a-3]}\right. \\
& \left.-\frac{1}{a \sqrt{4 a-1}[\sqrt{4 a-1}+\sqrt{2 a-2}]}\right\} \\
= & -\frac{2 a+1}{3 a[\sqrt{8 a-2}+\sqrt{12 a}]}-\frac{2 a(2 a+1)}{6(a-1) \sqrt{a-1}[2 \sqrt{4 a-1} \sqrt{2 a-2}+6 a-3]} \\
& -\frac{2 a+1}{6 \sqrt{a-1}}\left\{\frac{1}{(a-1)[2 \sqrt{4 a-1} \sqrt{2 a-2}+6 a-3]}-\frac{1}{a \sqrt{4 a-1}[\sqrt{4 a-1}+\sqrt{2 a-2}]}\right\} \\
= & -N_{1}-N_{2}-\frac{2 a+1}{6 \sqrt{a-1}} N_{3},
\end{aligned}
$$

where 


$$
\begin{aligned}
& N_{1}=\frac{2 a+1}{3 a[\sqrt{8 a-2}+\sqrt{12 a}]}>0, \\
& N_{2}=\frac{2 a(2 a+1)}{6(a-1) \sqrt{a-1}[2 \sqrt{4 a-1} \sqrt{2 a-2}+6 a-3]}>0 \text {, } \\
& N_{3}=\frac{1}{(a-1)[2 \sqrt{4 a-1} \sqrt{2 a-2}+6 a-3]}-\frac{1}{a \sqrt{4 a-1}[\sqrt{4 a-1}+\sqrt{2 a-2}]} \\
& =\frac{a \sqrt{4 a-1}[\sqrt{2 a-2}+\sqrt{4 a-1}]-(a-1)[2 \sqrt{4 a-1} \sqrt{2 a-2}+6 a-3]}{a(a-1) \sqrt{4 a-1}[2 \sqrt{4 a-1} \sqrt{2 a-2}+6 a-3][\sqrt{4 a-1}+\sqrt{2 a-2}]} \\
& =\frac{(2-a) \sqrt{2 a-2} \sqrt{4 a-1}+5-2(a-2)^{2}}{a(a-1) \sqrt{4 a-1}[2 \sqrt{4 a-1} \sqrt{2 a-2}+6 a-3][\sqrt{4 a-1}+\sqrt{2 a-2}]},
\end{aligned}
$$

since $5-2(a-2)^{2}>0$ for $a \in(1,2)$, hence $N_{3}>0$, then $N_{i}>0(i=1,2,3)$ and (42) imply that

$$
\lim _{t \rightarrow+\infty} E v_{2}(x, t)<0 \text {. }
$$

Therefore, we get the random perturbation of the traveling wave solution of (8) near the equilibrium point $(0,1)$ :

$$
\begin{aligned}
\left(\begin{array}{l}
u(x, t) \\
v(x, t)
\end{array}\right)=\left(\begin{array}{l}
0 \\
1
\end{array}\right)+\varepsilon\left(\begin{array}{l}
u_{1}(x, t) \\
v_{1}(x, t)
\end{array}\right)+\varepsilon^{2}\left(\begin{array}{l}
u_{2}(x, t) \\
v_{2}(x, t)
\end{array}\right), \\
\left(\begin{array}{l}
E u(x, t) \\
E v(x, t)
\end{array}\right)=\left(\begin{array}{l}
0 \\
1
\end{array}\right)+\varepsilon^{2}\left(\begin{array}{l}
E u_{2}(x, t) \\
E v_{2}(x, t)
\end{array}\right), \\
\lim _{t \rightarrow+\infty}\left(\begin{array}{l}
E u(x, t) \\
E v(x, t)
\end{array}\right)=\left(\begin{array}{l}
0 \\
1
\end{array}\right)+\varepsilon^{2} \lim _{t \rightarrow+\infty}\left(\begin{array}{l}
E u_{2}(x, t) \\
E v_{2}(x, t)
\end{array}\right),
\end{aligned}
$$

since

$$
\lim _{t \rightarrow+\infty} E u_{2}(x, t)>0, \quad \lim _{t \rightarrow+\infty} E v_{2}(x, t)<0,
$$

these imply that the effect of zero-mean white noise on the system near the lower equilibrium $u=0$ is to increase the expected value of $u(x, t)$ for all $x$, that is, the mean value $E u(x, t)$ is shifted above the equilibrium $u=0$. Similarly, near the upper equilibrium $v=1$ the white noise is to decrease the expected value of $v(x, t)$ for all $x$, that is, the mean value $\operatorname{Ev}(x, t)$ is shifted below the equilibrium $v=1$.

\section{Asymptotic Random Perturbations on the Right Stable State}

We now consider another equilibrium $(1,0)$ that is the right stead state of traveling wavefront of (2), i.e. $(U(+\infty), V(+\infty))=(1,0)$. Now we consider the equilibrium $\left(u_{0}, v_{0}\right)=(1,0)$. According to the condition (6), the linearized matrix of $(2)$ at $(1,0)$ is

$$
\boldsymbol{A}(1,0)=\left(\begin{array}{cc}
-1 & -a \\
0 & r(1-b)
\end{array}\right),
$$

it has two negative eigenvalues $\lambda_{1}=-1<0, \lambda_{2}=r(1-b)<0$, and there is an invertible matrix

$$
\boldsymbol{P}=\left(\begin{array}{cc}
1 & 1 \\
0 & \frac{1+a}{a}
\end{array}\right),
$$

such that 


$$
\boldsymbol{P}^{-1} \boldsymbol{A}(1,0) \boldsymbol{P}=\left(\begin{array}{cc}
-1 & 0 \\
0 & r(1-b)
\end{array}\right),
$$

thus

$$
\mathrm{e}^{A t}=\boldsymbol{P}\left(\begin{array}{cc}
\mathrm{e}^{-t} & 0 \\
0 & \mathrm{e}^{r(1-b) t}
\end{array}\right) \boldsymbol{P}^{-1}=\left(\begin{array}{cc}
\mathrm{e}^{-t} & \frac{a}{a+1}\left[\mathrm{e}^{r(1-b) t}-\mathrm{e}^{-t}\right] \\
0 & \mathrm{e}^{r(1-b) t}
\end{array}\right) .
$$

Therefore, the solution of (15) is

$$
\begin{aligned}
\left(\begin{array}{c}
u_{1}(x, t) \\
v_{1}(x, t)
\end{array}\right) & =\int_{0}^{t} \int_{-\infty}^{+\infty} G(x-y, t-s) \mathrm{e}^{A(1,0)(t-s)}\left(\begin{array}{l}
1 \\
0
\end{array}\right) w(\mathrm{~d} y, \mathrm{~d} s) \\
& =\int_{0}^{t} \int_{-\infty}^{+\infty} G(x-y, t-s)\left(\begin{array}{c}
\mathrm{e}^{-(t-s)} \\
0
\end{array}\right) w(\mathrm{~d} y, \mathrm{~d} s),
\end{aligned}
$$

so we have

$$
\begin{gathered}
E\left(\begin{array}{l}
u_{1}(x, t) \\
v_{1}(x, t)
\end{array}\right)=\left(\begin{array}{l}
0 \\
0
\end{array}\right), \text { and } v_{1}(x, t) \equiv 0, \\
E u_{1}^{2}(x, t)=E\left(\int_{0}^{t} \int_{-\infty}^{+\infty} G(x-y, t-s) \mathrm{e}^{-(t-s)} w(\mathrm{~d} y, \mathrm{~d} s)\right)^{2} \\
=\int_{0}^{t} \int_{-\infty}^{+\infty} G^{2}(x-y, t-s) \mathrm{e}^{-2(t-s)} \mathrm{d} y \mathrm{~d} s \\
=\int_{0}^{t} \frac{1}{\sqrt{8 \pi(t-s)}} \mathrm{e}^{-2(t-s)} \mathrm{d} s=\int_{0}^{t} \frac{1}{\sqrt{8 \pi s}} \mathrm{e}^{-2 s} \mathrm{~d} s .
\end{gathered}
$$

The solution of (16) is

$$
\begin{aligned}
\left(\begin{array}{c}
u_{2}(x, t) \\
v_{2}(x, t)
\end{array}\right) & =-\int_{0}^{t} \int_{-\infty}^{+\infty} G(x-y, t-s) \mathrm{e}^{A(t-s)}\left(\begin{array}{c}
\left(u_{1}^{2}+a u_{1} v_{1}\right)(y, s) \\
\left(b r u_{1} v_{1}+r v_{1}^{2}\right)(y, s)
\end{array}\right) \mathrm{d} y \mathrm{~d} s \\
& =-\int_{0}^{t} \int_{-\infty}^{+\infty} G(x-y, t-s) \mathrm{e}^{A(t-s)}\left(\begin{array}{c}
u_{1}^{2}(y, s) \\
0
\end{array}\right) \mathrm{d} y \mathrm{~d} s \\
& =-\int_{0}^{t} \int_{-\infty}^{+\infty} G(x-y, t-s)\left(\begin{array}{c}
\mathrm{e}^{-(t-s)} u_{1}^{2}(y, s) \\
0
\end{array}\right) \mathrm{d} y \mathrm{~d} s,
\end{aligned}
$$

hence we have $v_{2}(x, t) \equiv 0, E v_{2}(x, t)=0$ and

$$
\begin{aligned}
E u_{2}(x, t) & =-\int_{0}^{t} \int_{-\infty}^{+\infty} G(x-y, t-s) \mathrm{e}^{-(t-s)} u_{1}^{2}(y, s) \mathrm{d} y \mathrm{~d} s \\
& =-\int_{0}^{t} \mathrm{e}^{-(t-s)} E u_{1}^{2}(y, s) \mathrm{d} s=-\int_{0}^{t} \mathrm{e}^{-(t-s)} \int_{0}^{s} \frac{1}{\sqrt{8 \pi \tau}} \mathrm{e}^{-2 \tau} \mathrm{d} \tau \mathrm{d} s .
\end{aligned}
$$

Let $t \rightarrow+\infty$, we have

$$
\begin{aligned}
\lim _{t \rightarrow+\infty} E u_{2}(x, t) & =-\lim _{t \rightarrow+\infty} \int_{0}^{t} \mathrm{e}^{-(t-s)} \int_{0}^{s} \frac{1}{\sqrt{8 \pi \tau}} \mathrm{e}^{-2 \tau} \mathrm{d} \tau \mathrm{d} s=-\lim _{t \rightarrow+\infty} \frac{\int_{0}^{t} \mathrm{e}^{s} \int_{0}^{s} \frac{1}{\sqrt{8 \pi \tau}} \mathrm{e}^{-2 \tau} \mathrm{d} \tau \mathrm{d} s}{\mathrm{e}^{t}} \\
& =-\int_{0}^{+\infty} \frac{1}{\sqrt{8 \pi \tau}} \mathrm{e}^{-2 \tau} \mathrm{d} \tau \mathrm{d} s=-\frac{1}{\sqrt{8 \pi}} \sqrt{\frac{\pi}{2}}=-\frac{1}{4}<0 .
\end{aligned}
$$

Then, we get the random perturbation of the traveling wavefront of (8) near the equilibrium point $(1,0)$ : 


$$
\begin{gathered}
\left(\begin{array}{l}
u(x, t) \\
v(x, t)
\end{array}\right)=\left(\begin{array}{l}
1 \\
0
\end{array}\right)+\varepsilon\left(\begin{array}{c}
u_{1}(x, t) \\
0
\end{array}\right)+\varepsilon^{2}\left(\begin{array}{c}
u_{2}(x, t) \\
0
\end{array}\right)+o\left(\varepsilon^{2}\right), \\
\left(\begin{array}{l}
E u(x, t) \\
E v(x, t)
\end{array}\right)=\left(\begin{array}{l}
1 \\
0
\end{array}\right)+\varepsilon^{2}\left(\begin{array}{c}
E u_{2}(x, t) \\
0
\end{array}\right)+o\left(\varepsilon^{2}\right), \\
\lim _{t \rightarrow+\infty}\left(\begin{array}{l}
E u(x, t) \\
E v(x, t)
\end{array}\right)=\left(\begin{array}{l}
1 \\
0
\end{array}\right)+\varepsilon^{2} \lim _{t \rightarrow+\infty}\left(\begin{array}{c}
E u_{2}(x, t) \\
0
\end{array}\right)+o\left(\varepsilon^{2}\right) .
\end{gathered}
$$

From (56),

$$
\lim _{t \rightarrow+\infty} E u_{2}(x, t)=-\frac{1}{4}<0
$$

implies that the effect of zero-mean white noise on the system near the lower equilibrium $u=1$ is to decrease the expected value of $u(x, t)$ for all $x$, that is, the mean value $E u(x, t)$ is shifted below the equilibrium $u=1$. On the other hand, $v_{1}(x, t) \equiv 0$ and $v_{2}(x, t) \equiv 0$ imply that the random perturbations do not alter the mean value $\operatorname{Ev}(x, t)$ near the lower equilibrium $v=0$ for all $x$, in fact $E v(x, t) \equiv 0$.

Remark 1. In the future paper, we will consider simulation of solutions on bounded domains and compare with the present analytical results. Also, we want to consider the system that the white noise is included in the 2nd component of (8), but according to the complicated calculations in Sections 3 and 4, we must look for a new idea to deal with this coupled problem.

\section{Acknowledgements}

This work was supported by National Natural Sciences Foundation of China (Grant No. 11471129). Corresponding author: Yanbin Tang.

\section{References}

[1] Smoller, J. (1983) Shock Waves and Reaction Diffusion Equations. Springer, New York. http://dx.doi.org/10.1007/978-1-4684-0152-3

[2] Aronson, D.G. and Weinberger, H.F. (1975) Nonlinear Diffusion in Population Genetics, Combustion and Nerve Propagation. Lecture Notes in Mathematics, 446, 5-49. http://dx.doi.org/10.1007/BFb0070595

[3] Fisher, R.A. (1937) The Wave of Advance of Advantageous Genes. Annals of Eugenics, 7, 355-369. http://dx.doi.org/10.1111/j.1469-1809.1937.tb02153.x

[4] Kolmogorov, A.N., Petrovsky, I.G. and Piskunov, N.S. (1937) Investigation of the Equation of Diffusion Combined with Increasing of the Substance and Its Application to a Biology Problem. Bulletin of Moscow State University Series A: Mathematics and Mechanics, 1, 1-25.

[5] Zeldovich, Y.B., Barenblatt, G.I., Librovich, V.B. and Makhviladze, G.M. (1983) Mathematical Theory of Combustion and Explosions. Consultants Bureau, New York.

[6] Volpert, V. and Petrovskii, S. (2009) Reaction-Diffusion Waves in Biology. Physics of Life Reviews, 6, 267-310. http://dx.doi.org/10.1016/j.plrev.2009.10.002

[7] Tuckwell, H.C. (1993) Random Fluctuations at an Equilibrium of a Nonlinear Reaction Diffusion Equation. Applied Mathematics Letters, 6, 79-81. http://dx.doi.org/10.1016/0893-9659(93)90017-H

[8] Britton, N.F. (1986) Reaction-Diffusion Equations and Their Applications to Biology. Academic Press, New York.

[9] Tang, Y.B. and Zhou, L. (2007) Stability Switch and Hopf Bifurcation for a Diffusive Prey Predator System with Delay. Journal of Mathematical Analysis and Applications, 334, 1290-1307. http://dx.doi.org/10.1016/j.jmaa.2007.01.041

[10] Tang, Y.B. and Wang, J.L. (2009) Bifurcation Analysis on a Reactor Model with Combination of Quadratic and Cubic steps. Journal of Mathematical Chemistry, 46, 1394-1408. http://dx.doi.org/10.1007/s10910-009-9523-7

[11] Vilar, J.M.G. and Rubi, J.M. (1997) Spatiotemporal Stochastic Resonance in the Swift-Hohenberg Equation. Physical Review Letters, 78, 2886-2889. http://dx.doi.org/10.1103/PhysRevLett.78.2886

[12] Vilar, J.M.G. and Solé, R.V. (1998) Effects of Noise in Symmetric Two-Species Competition. Physical Review Letters, 80, 4099-4102. http://dx.doi.org/10.1103/PhysRevLett.80.4099

[13] Zhou, L., Tang, Y.B. and Hussein, S. (2002) Stability and Hopf Bifurcation for a Delay Competition Diffusion System. 
Chaos, Solitons \& Fractals, 14, 1201-1225. http://dx.doi.org/10.1016/S0960-0779(02)00068-1

[14] Zhu, C. and Yin, G. (2009) On Competitive Lotka-Volterra Model in Random Environments. Journal of Mathematical Analysis and Applications, 357, 154-170. http://dx.doi.org/10.1016/j.jmaa.2009.03.066

[15] Fei, N. and Carr, J. (2003) Existence of Traveling Waves with Their Minimal Speed for a Diffusing Lotka-Volterra System. Nonlinear Analysis: Real World Applications, 4, 503-524. http://dx.doi.org/10.1016/S1468-1218(02)00077-9

[16] Conley, C. and Gardner, R. (1984) An Application of the Generalized Morse Index to Travelling Wave Solutions of a Competitive Reaction-Diffusion Model. Indiana University Mathematics Journal, 33, 319-343. http://dx.doi.org/10.1512/iumj.1984.33.33018

[17] Gardner, R. (1982) Existence and Stability of Traveling Wave Solutions of Competition Models: A Degree Theoretic Approach. Journal of Differential Equations, 44, 343-364. http://dx.doi.org/10.1016/0022-0396(82)90001-8

[18] Tang, M. and Fife, P. (1980) Propagating Fronts for Competing Species Equations with Diffusion. Archive for Rational Mechanics and Analysis, 73, 69-77. http://dx.doi.org/10.1007/BF00283257

[19] Kanel, J.I. and Zhou, L. (1996) Existence of Wave Front Solutions and Estimates of Wave Speed for a CompetitionDiffusion System. Nonlinear Analysis, 27, 579-587. http://dx.doi.org/10.1016/0362-546X(95)00221-G

[20] Bao, X.X. and Wang, Z.C. (2013) Existence and Stability of Time Periodic Traveling Waves for a Periodic Bistable Lotka-Volterra Competition System. Journal of Differential Equations, 255, 2402-2435. http://dx.doi.org/10.1016/j.jde.2013.06.024

[21] Tuckwell, H.C. (2008) Nonlinear Effects in White-Noise Driven Spatial Diffusion: General Analytical Results and Probabilities of Exceeding Threshold. Physica A, 387, 1455-1463. http://dx.doi.org/10.1016/j.physa.2007.10.062

[22] Wu, E.Z. and Tang, Y.B. (2012) Random Perturbations of Reaction-Diffusion Waves in Biology. Wave Motion, 49, 632-637. http://dx.doi.org/10.1016/j.wavemoti.2012.04.004 